\title{
Meilleure estimation du fardeau que représentent les facteurs de risque de maladie chronique pour la santé et l'économie au Manitoba
}

H. Krueger, Ph. D. (1, 2); D. Williams, M. Sc. (2); A. E. Ready, Ph. D. (3); L. Trenaman, B. Sc. (2); D. Turner, Ph. D. $(4,5)$

Cet article a fait l'objet d'une évaluation par les pairs.

\section{Résumé}

Introduction : L'estimation du fardeau global que représentent les facteurs de risque multiples au sein d'une population présente certains défis d'ordre analytique. Nous décrivons une méthodologie permettant de tenir compte des facteurs de risque se chevauchant dans certaines sous-populations et entraînant un " double compte » des maladies et du fardeau économique qu'ils engendrent.

Méthodologie : Notre démarche permet d'analyser avec précision le fardeau économique global des maladies chroniques dans un cadre multifactoriel tout en tenant compte de l'incidence du poids en tant qu'exposition continue ou polytomique (allant de l'absence d'excédent de poids au surpoids et à l'obésité). Nous appliquons cette méthode au tabagisme, à l'inactivité physique et au surpoids et à l'obésité à la province du Manitoba (Canada).

Résultats : En 2008, le fardeau économique global annuel des facteurs de risque au Manitoba était d'environ 1,6 milliard de dollars (557 millions pour le tabagisme, 299 millions pour l'inactivité physique et 747 millions pour le surpoids et l'obésité). Le fardeau total représente un rajustement à la baisse de 12,6 \% lorsqu'on tient compte de l'effet des facteurs de risque multiples chez certaines personnes.

Conclusion : Une meilleure estimation du fardeau économique global des facteurs de risque multiples au sein d'une population peut faciliter l'établissement des priorités et améliorer le soutien aux initiatives de prévention primaire.

Mots-clés : fraction étiologique du risque, facteurs de risque, obésité, inactivité physique, tabagisme, maladie chronique

\section{Introduction}

Les planificateurs de soins de santé se préoccupent depuis longtemps de la " transition épidémiologique ", soit le processus par lequel les maladies chroniques en viennent à supplanter les infections pandémiques en tant que principale source de morbidité et de mortalité au monde ${ }^{1}$. La dernière phase de cette transi- tion est marquée par une prévalence accrue du surpoids et de l'obésité ainsi que de l'inactivité physique dans de nombreux pays $^{2}$. Un excédent de poids ou l'inactivité physique, ou les deux, ont un rôle à jouer dans l'apparition des maladies chroniques, par exemple les maladies cardiovasculaires, les accidents vasculaires cérébraux (AVC), le diabète de type 2 , les maladies rénales chroniques, l'arthrose et certains cancers $^{3-12}$. Ces facteurs de risque s'ajoutent donc au tabagisme ${ }^{13}$ comme principales cibles de prévention.

Le fardeau économique qu'engendrent ces facteurs de risque a fait l'objet de calculs estimatifs dans de nombreux pays du monde ${ }^{14}$, dont le Canada dans son ensemble ${ }^{15-19}$ et quelques-unes de ses provinces $^{20,21}$. Il est souvent pertinent, en plus de connaître les coûts liés à un facteur de risque (par exemple le tabagisme), d'évaluer le fardeau économique global de deux facteurs de risque ou plus au sein d'une population. Cette donnée peut faciliter l'adoption de stratégies de prévention visant plus d'un facteur de risque, par exemple la mise en place de programmes de santé publique qui portent à la fois sur l'inactivité physique et le surpoids et l'obésité. L'estimation du fardeau global que représentent les facteurs de risque multiples au sein d'une population présente cependant certains défis d'ordre analytique ${ }^{22}$. Certains coûts (comme ceux liés aux nouveaux cas de maladie ou à la mort) ne sont, par définition, comptabilisés qu'une seule fois. Il est donc important de tenir compte de l'effet combiné des facteurs de risque multiples chez une même personne, et surtout de corriger toute hausse du fardeau économique calculé en raison du double compte des cas et des coûts.

La fraction étiologique du risque (FER) offre un excellent moyen d'interpréter les causes sous l'angle pratique de la prévention. Pour simplifier, la FER est la propor-

Rattachement des auteurs :

1. École de santé publique et de santé des populations, Université de la Colombie-Britannique, Vancouver (Colombie-Britannique), Canada

2. H. Krueger \& Associates Inc., Delta (Colombie-Britannique), Canada

3. Faculté de kinésiologie et de gestion des loisirs, Université du Manitoba, Winnipeg (Manitoba), Canada

4. Département des sciences de la santé communautaire, Université du Manitoba, Winnipeg (Manitoba), Canada

5. Action cancer Manitoba, Winnipeg (Manitoba), Canada

Correspondance : Hans Krueger, H. Krueger \& Associates Inc., 4554 48B Street, Delta (Colombie-Britannique) V4K 2R8; tél. : 604-946-5464; courriel : hans@krueger.ca 
tion des conséquences (coûts) des maladies qui sera éliminée si l'exposition aux facteurs de risque qui en sont la cause est supprimée. Cette technique se complique lorsque le but est d'évaluer l'effet combiné de facteurs de risque multiples.

Un certain nombre d'approches innovatrices ont cependant été élaborées afin de quantifier les conséquences des facteurs de risque multiples au sein de cohortes précises $^{23}$. Ainsi, le World Cancer Research Fund a eu recours à un procédé appelé "prévention séquentielle », qu’il définit ainsi ${ }^{24, \text { p. } 149}$ :

[Traduction] Comme aucun cas individuel de cancer ne peut être prévenu plus d'une fois, le calcul a été fait de façon à éviter la possibilité du «double compte». La FER pour la première exposition a donc été soustraite de $100 \%$, alors que la FER de la seconde exposition a été appliquée au reste. Ce procédé a été suivi de façon séquentielle pour toutes les expositions pertinentes, ce qui a donné une FER estimative pour toutes les expositions combinées.

Bien qu'il soit logique de tenir compte des facteurs de risque qui se chevauchent, le travail à faire pour démêler l'incidence de ces facteurs ne fait souvent pas partie des estimations du fardeau économique qui s'y rattache. À titre d'exemple concret, mentionnons une série d'articles publiés entre 2005 et 2009 par un groupe de la British Heart Foundation concernant le fardeau que représente pour la GrandeBretagne un mauvais état de santé en raison de l'inactivité physique ${ }^{25}$, d'un surpoids ou d'une obésité ${ }^{26}$, du tabagisme $^{27}$ et d'autres facteurs de risques $^{28,29}$. Dans un article de synthèse, les auteurs reconnaissent le fait que « [traduction] le chevauchement possible des facteurs de risque (comme le surpoids et l'obésité) n’a pas été examiné ici, mais il devrait être pris en considération au moment de calculer le fardeau économique total de ces facteurs de risque ${ }^{30, \text { p. } 534}$. »

Pour remédier à la situation, nous présentons ici une méthodologie permettant de tenir compte des facteurs de risque qui se chevauchent au moment d'estimer le fardeau économique global des maladies chroniques associées. Notre démarche compte quatre étapes : 1) considérer le rôle du poids en tant qu'exposition continue ou "polytomique » selon qu'il y ait absence d'excédent de poids, surpoids ou obésité; 2) estimer le fardeau global des maladies chroniques dans un cadre multifactoriel de façon à tenir compte de l'effet de plus d'un facteur de risque; 3) estimer le fardeau économique global rajusté en fonction de la présence de facteurs de risque multiples chez certaines personnes; et 4) ventiler le fardeau total afin d'obtenir une estimation du coût économique lié par déduction à chaque facteur de risque.

À notre connaissance, notre article est la première publication sur la question du double compte des coûts attribuable aux facteurs de risque qui se chevauchent chez certaines personnes dans le cadre de l'estimation du fardeau économique des facteurs de risque multiples.

Pour illustrer l'utilité de cette démarche, nous avons estimé le fardeau économique des maladies attribuables au tabagisme, à l'inactivité physique et au surpoids et à l'obésité pour le Manitoba. Cette province compte environ 1,2 million d'habitants ${ }^{31}$. Malgré une économie fondée sur une agriculture solide et sur l'exploitation des ressources naturelles, environ $60 \%$ des Manitobains vivent dans la capitale provinciale, Winnipeg. Le Manitoba abrite également un grand nombre de membres des Premières nations (11 \% de la population provinciale) $)^{32}$.

\section{Méthodologie}

Nous avons suivi une démarche fondée sur la FER pour estimer le fardeau économique lié aux divers facteurs de risque. Pour simplifier, disons que la FER renvoie à la proportion des conséquences des maladies qui sont générées au sein d'une population par un facteur de risque en particulier ${ }^{33}$. Pour obtenir les résultats dont nous faisons rapport dans cet article, il a fallu calculer une FER spécifique au Manitoba pour chacune des maladies associées aux facteurs de risque pertinents, puis combiner cette donnée à l'estimation des coûts spécifiques au Manitoba pour traiter ces maladies et faire face aux conséquences indirectes de la mortalité et de la morbidité.
La FER est une mesure statistique qui combine deux facettes d'un facteur de risque et de ses conséquences sur la maladie : le risque relatif (RR) du facteur de risque en lien avec une maladie en particulier et la prévalence de l'exposition au facteur de risque au sein de la population concernée.

\section{Risque relatif}

Les risques relatifs (RR) liés à l'inactivité physique proviennent des méta-analyses de Katzmarzyk et Janssen ${ }^{16}$. La majorité des études mentionnées dans l'article de Katzmarzyk et Janssen ${ }^{16}$ ont intégré un indice d'obésité à l'analyse pour que les effets de l'activité physique sur le risque de maladie puissent être considérés comme indépendants de l’obésité. Les méta-analyses de Guh et collab. sont la source des RR liés au surpoids et à l'obésité ${ }^{34}$. Les auteurs n'ont pas tenu compte de l'inactivité physique comme facteur de risque potentiellement confusionnel, puisque « [traduction] l'inactivité physique est souvent très mal documentée, et son inclusion aurait eu pour effet de réduire le nombre d'études analysées » ${ }^{34, \text { p. } 15}$.

Nous avons consulté deux sources pour réunir les $\mathrm{RR}$ de maladies attribuables au tabagisme. Un article publié en 2008 par Gandini et collab. ${ }^{35}$ offre une méta-analyse détaillée sur les cancers liés à l'usage du tabac, notamment des RR rajustés en fonction des facteurs de confusion connus (cancers de l'œsophage et du tube digestif supérieur pour la consommation d'alcool, cancer de l'estomac pour l'alimentation, cancer du foie pour l'infection à l'hépatite B ou C, cancer du col de l'utérus pour l'infection au virus du papillome humain et cancer du rein pour l'indice de masse corporelle $)^{35}$. Il convient de noter que le tabagisme n'est plus un facteur de risque important pour les cancers du foie ou du col de l'utérus après ces rajustements. Le $\mathrm{RR}$ de maladies cardiovasculaires et respiratoires est tiré d'une publication de Thun et collab. ${ }^{36}$, lesquels ont rajusté tous les RR en fonction de l'âge, de la race, de la scolarité, de l'état matrimonial, de l'emploi, de la consommation de fruits et de légumes, de l'utilisation d'aspirine, de la consommation d'alcool, de l'indice de masse corporelle (IMC), de l'activité 
physique et de la consommation d'aliments gras. Enfin, le RR de pneumonie, d'influenza, de bronchite et d'emphysème a été rajusté en fonction de l'exposition professionnelle à l'amiante.

La plupart des sources, à l'exception de celles portant sur l'inactivité physique, présentaient des données sur le RR selon le sexe. Un examen plus approfondi des études pour cerner les variations de l'inactivité physique selon le sexe permet d'affirmer que le RR ne diffère pas énormément entre les hommes et les femmes pour ce facteur de risque $\mathrm{e}^{37,38,39}$.

Les estimations ponctuelles des RR servent aux calculs dans le modèle de base, tandis que les limites supérieures et inférieures de l'intervalle de confiance (IC) à $95 \%$ sont utilisées dans le cadre de l'analyse de sensibilité.

\section{Exposition aux facteurs de risque}

L'autre moitié du calcul de la FER repose sur la disponibilité de données de qualité relativement à la prévalence des facteurs de risque $^{40}$. L'analyse de l'exposition de la population manitobaine au tabagisme, à l'inactivité physique et au surpoids et à l'obésité a débuté par les données tirées de l'Enquête sur la santé dans les collectivités canadiennes (ESCC) de 2008. Les données sur le tabagisme incluaient tous les « fumeurs actuels » (ceux qui fument tous les jours et à l'occasion), celles sur le surpoids et l'obésité incluaient les personnes dont l'IMC se situait entre $25 \mathrm{~kg} / \mathrm{m}^{2}$ et $30 \mathrm{~kg} / \mathrm{m}^{2}$ pour le surpoids et à $30 \mathrm{~kg} / \mathrm{m}^{2}$ et plus pour l'obésité (selon la taille et le poids autodéclarés), alors que celles sur l'inactivité physique incluaient les personnes classées dans l'ESCC comme « inactives".

Nous avons apporté plusieurs corrections aux données de base de l'ESCC afin de combler ses lacunes connues. D'abord, nous avons utilisé les données de l'Enquête sur la santé des jeunes au Manitoba (ESJM) pour faire des rajustements en fonction de l'usage du tabac et de l'inactivité physique chez les jeunes ${ }^{41}$. Les données tirées de l'ESCC conduisent à penser qu'environ $10 \%$ des jeunes Manitobains âgés de 12 à 19 ans étaient fumeurs en 2008, contre $21,2 \%$ des jeunes de la $6^{\mathrm{e}}$ à la $12^{\mathrm{e}}$ année d'après l'ESJM. Par ailleurs, la prévalence des jeunes physiquement inactifs était de $32 \%$ dans l'ESCC, contre 19,3 \% dans l'ESJM.

Ensuite, nous avons estimé les taux d'inactivité physique chez les enfants de moins de 12 ans selon les taux de l'ESJM $(16,4 \%$ pour les garçons et $22,1 \%$ pour les filles). Les taux de surpoids et d'obésité chez les enfants et les adolescents de moins de 18 ans ont été estimés en fonction des taux spécifiques au Manitoba dans l'ESCC pour les 20-34 ans (surpoids : $34,5 \%$ chez les garçons et 36,6 \% chez les filles; obésité : 15,6\% chez les garçons et $14,7 \%$ chez les filles) ${ }^{42}$. Bien que l'ESCC fournisse une estimation des taux de surpoids et d'obésité combinés pour les jeunes de 12 à 19 ans, les résultats présentent un coefficient de variation élevé et doivent être utilisés avec circonspection ${ }^{42}$. En outre, les comportements liés à l'obésité - en particulier l'inactivité physique et la mauvaise alimentation - qui ont été adoptés dans l'enfance ont tendance à se poursuivre à l'âge adulte ${ }^{43}$.

Finalement, l'ESCC ne tient pas compte des personnes vivant dans les réserves autochtones, lesquelles représentent environ 55000 Manitobains ${ }^{44}$. Nous avons donc utilisé les résultats de l'Enquête régionale longitudinale sur la santé des Premières nations de 2002-2003 pour cerner et rajuster la prévalence élevée du tabagisme (62\%) et du surpoids et de l'obésité (75\%) parmi les adultes de 18 ans et plus et pour toute la population vivant dans une réserve ${ }^{45}$.

Un dernier rajustement a été inspiré des travaux d'Anis et collab. ${ }^{18}$, qui ont utilisé la prévalence du tour de taille plutôt que l'IMC pour certaines catégories de maladies, dont la cardiopathie ischémique, l'hypertension, le diabète de type 2 et les maladies de la vésicule biliaire.

\section{Niveaux d'exposition multiples}

La version simplifiée du calcul de la FER, dérivée de la prévalence d'un seul facteur de risque et du RR de la maladie qui y est associée, a recours à la formule $(E(R R-1)) /(E(R R-1)+1)$, où $E$ est la proportion de la population exposée au facteur concerné et RR est le risque relatif de l'apparition de la maladie dans le groupe exposé au facteur.

Cela dit, il faut employer une version plus complexe pour calculer la FER en présence d'un facteur de risque polytomique, c'està-dire à facettes multiples. C'est le cas du surpoids et de l'obésité : ces deux catégories biologiques font partie du même continuum. Il ne faut donc pas calculer algébriquement le FER de base pour le surpoids et celui pour l'obésité, puis simplement additionner les deux chiffres pour en tirer une FER globale de l'exposition à un excédent de poids. Il faut plutôt concevoir le surpoids et l'obésité comme une exposition trichotome à l'excédent de poids, avec les catégories d'exposition suivantes : 1) aucun excédent de poids, 2) excédent moyen de poids ou surpoids (prévalence $\mathrm{E}_{\mathrm{OW}}$ ) et 3 ) excédent de poids important ou obésité (prévalence $\mathrm{E}_{\mathrm{OB}}$ ). Le calcul de la FER est le suivant ${ }^{46}$ :

$$
\begin{aligned}
& \mathrm{E}_{\mathrm{OW}}\left(\mathrm{RR}_{\mathrm{OW}}-1\right)+\mathrm{E}_{\mathrm{OB}}\left(\mathrm{RR}_{\mathrm{OB}}-1\right) / \\
& \mathrm{E}_{\mathrm{OW}}\left(\mathrm{RR}_{\mathrm{OW}}-1\right)+\mathrm{E}_{\mathrm{OB}}\left(\mathrm{RR}_{\mathrm{OB}}-1\right)+1
\end{aligned}
$$

\section{Facteurs de risque multiples}

Lorsque l'ensemble des données sur l'exposition à des facteurs de risques multiples (p. ex., tabagisme et surpoids ou obésité chez la même personne) et sur le RR lié à chaque ensemble de causes est connu, il est alors facile de calculer la FER pour une combinaison de risques. Cependant, lorsqu'il manque des données sur les facteurs de risque qui se chevauchent, comme c'est souvent le cas, il est important, là aussi, d'éviter de simplement additionner les FER de base pour chaque facteur de risque afin d'obtenir une FER combinée pour le cadre multifactoriel. Il faut plutôt estimer de manière plus précise la FER d'un cadre multifactoriel en utilisant l'équation suivante $^{47}$ :

$$
1-\left[\left(1-\mathrm{FER}_{1}\right)\left(1-\mathrm{FER}_{2}\right)\left(1-\mathrm{FER}_{3}\right)\right]
$$

où $\mathrm{FER}_{1}$ renvoie à la $\mathrm{FER}$ liée au premier facteur de risque, et ainsi de suite. 
Cette équation permet un calcul plus précis en présence de deux conditions : 1) les facteurs de risque présents sont statistiquement indépendants (p. ex. le fait d'en vivre un ne rend pas la personne plus ou moins susceptible de vivre l'autre, ou le regroupement des facteurs de risque est limité) et 2) leurs effets combinés sont multiplicatifs (synergiques). Il est possible de démontrer que ces deux conditions s'appliquent très bien à une situation mettant en jeu l'obésité et le tabagisme $^{48,49}$ et s'appliquent assez bien à l'obésité et à l'inactivité physique ${ }^{50,51}$. Des enquêtes équivalentes portant sur le tabagisme combiné à l'inactivité sont rares.

Cette équation de rajustement peut être appliquée à d'autres facteurs de risque, ainsi qu'aux aspects du développement de la maladie qui vont au-delà de l'incidence de base, notamment aux taux de mortalité et d'invalidité. Dans le cadre de cette analyse, nous avons eu recours à l'équation de rajustement pour générer une FER plus précise en ce qui concerne les coûts directs de la maladie.

\section{Coûts directs}

Nous avons estimé le fardeau économique (coûts directs et indirects) des facteurs de risque au Manitoba avec la méthode du coût de la maladie fondée sur la prévalence $^{52}$ et l'avons exprimé en dollars canadiens de 2008 .

Nous avons entrepris le calcul des coûts directs à l'aide de la méthode adoptée par Anis et collab. ${ }^{18}$. En résumé, les coûts directs, qui comprennent les soins hospitaliers, les services médicaux, les autres services professionnels des soins de santé (à l'exclusion des services dentaires), les médicaments, la recherche en santé et les " autres » dépenses en santé, ont été extraits de la Base de données sur les dépenses nationales de santé pour le Manitoba ${ }^{53}$. Les coûts, à l'exception des soins hospitaliers, ont été attribués à chacune des catégories de comorbidité en fonction des poids publiés dans le rapport Le fardeau économique de la maladie au Canada $1998^{54}$. Les frais hospitaliers ont été attribués à chaque comorbidité en fonction de la proportion du total de jours-patient (selon les données tirées de la Base de données sur la morbidité hospita- lière de l'Institut canadien d'information sur la santé $2000-2001^{55}$ ) utilisés pour traiter les patients présentant une comorbidité au Manitoba. Les coûts directs estimatifs totaux ont été répartis entre les hommes et les femmes en fonction de la proportion des jours-patient passés à l'hôpital en 20002001 par les hommes et par les femmes pour chacune des comorbidités. Enfin, les coûts de chaque comorbidité selon le sexe ont été multipliés par la FER calculée pour le sexe et la comorbidité au Manitoba.

\section{Rajustement des coûts directs dans un cadre multifactoriel}

Nous avons ensuite appliqué la formule présentée précédemment pour calculer la FER combinée dans un cadre multifactoriel aux coûts directs bruts calculés attribuables à chacun des facteurs de risque que sont le tabagisme, le surpoids ou l'obésité et l'inactivité physique. Les coûts directs bruts pour chaque facteur de risque ont été incorporés à la formule de rajustement (soit $\mathrm{FER}_{1}=$ FER brute du coût du tabagisme, etc.) afin de générer une FER rajustée des coûts directs dans le cadre multifactoriel. Cette technique a permis de réduire les coûts directs combinés de 12,6\% (les faisant passer de 560,8 à 490,3 millions de dollars par année).

Ayant déterminé le plus rigoureusement possible l'effet combiné sur la population de la présence de facteurs de risque multiples, il est toujours utile, pour établir avec une grande précision les priorités en matière de prévention, préparer les messages éducatifs destinés au public, etc., d'avoir une meilleure idée de l'effet approximatif d'un facteur de risque en particulier. Nous avons donc ajouté une étape de ventilation à la fin du processus d'établissement des coûts directs afin d'attribuer par déduction un fardeau économique à chaque facteur de risque. Pour ce faire, nous sommes revenus aux coûts bruts pour chaque facteur de risque, puis nous avons divisé chacun de ces chiffres par leur somme (soit le coût total brut des facteurs de risque combinés) pour ainsi obtenir une proportion. Ce rapport a ensuite été appliqué aux coûts directs totaux rajustés, ce qui a donné le fardeau économique rajusté, ventilé selon la maladie et attribuable (par déduction) à chaque facteur de risque.

\section{Coûts indirects}

Nous avons calculé les coûts indirects (mortalité prématurée, invalidité à court et à long terme) en suivant la méthode utilisée dans l'enquête FEMC 1998 (méthode du capital humain modifiée) ${ }^{54}$.

Voici, plus précisément, les étapes suivies pour estimer les coûts indirects :

1. Six catégories de diagnostics utilisées dans l'enquête FEMC 1998 ont été sélectionnées pour couvrir les comorbidités/maladies pertinentes, puis les coûts directs et indirects de ces six catégories ont été extraits.

2. Ces données ont servi à établir un rapport entre les coûts directs et indirects de chacune des catégories de diagnostics, stratifié en fonction de la catégorie spécifique du coût indirect. Par exemple, les coûts indirects liés au cancer sont 4,6 fois (459\%) plus élevés que les coûts directs, et surtout attribuables à la mortalité prématurée. À l'inverse, les coûts indirects liés aux maladies musculosquelettiques sont 5,2 fois (519\%) plus élevés que les coûts directs et, dans ce cas, la grande majorité des coûts plus élevés est liée à l'invalidité à long terme plutôt qu'au décès prématuré (voir le tableau 1).

3. Les rapports pertinents (selon la catégorie de diagnostic et la catégorie spécifique du coût indirect) ont ensuite été appliqués aux coûts directs déterminés précédemment, attribuables à chaque facteur de risque et rajustés en fonction du cadre multifactoriel afin d'obtenir des données sur le coût indirect équivalentes.

Une description détaillée des étapes suivies dans le cadre de cette analyse, exemples à l'appui, est disponible sur demande.

\section{Résultats}

Le tableau 2 présente la prévalence entièrement rajustée de l'exposition aux facteurs de risque, les données statistiquement significatives sur le RR selon le sexe, ainsi que la FER calculée de l'incidence de la maladie liée à chaque facteur de risque. 
TABLEAU 1

Fardeau économique de la maladie au Canada selon la catégorie de diagnostic

\begin{tabular}{lcccc} 
Catégorie de diaǵnostic & \multicolumn{3}{c}{ Coûts indirects en tant que pourcentages des coûts directs, Canada, 1998 } \\
& Mortalité (\%) & Invalidité à long terme (\%) & Invalidité à court terme (\%) & Total des coûts indirects (\%) \\
\hline Cancer & 415 & 38 & 7 & 459 \\
Maladies cardiovasculaires & 121 & 46 & 28 & 70 \\
Maladies respiratoires & 48 & 51 & 3 & 171 \\
Maladies endocriniennes et connexes & 64 & 14 & 20 & 65 \\
Maladies du tube digestif & 32 & 476 & 38 & 519 \\
Maladies musculosquelettiques & 5 & & \\
\hline
\end{tabular}

La FER pour toutes les comorbidités, à l'exception évidente des cancers gynécologiques et du sein, varie selon le sexe. Par exemple, 38,8\% des cas de diabète de type 2 sont attribuables au Manitoba à l'obésité chez les hommes, tandis que cette proportion est de 48,2\% chez les femmes, et ce, malgré la prévalence plus élevée d'obésité chez les Manitobains (19,8 \%) que chez les Manitobaines (18,7 \%). La FER globale plus élevée chez les femmes s'explique par un RR beaucoup plus élevé $(12,41)$ que celui des hommes $(6,74)$ pour le diabète de type 2. Ce type d'analyse détaillée a d'importantes répercussions sur l'établissement des coûts directs et indirects.

Le tableau 3 présente une synthèse des estimations rajustées de la prévalence des facteurs de risque de maladies chroniques, le nombre absolu de Manitobains exposés à chaque facteur de risque et les résultats entièrement rajustés de l'analyse du fardeau économique. En 2008, au Manitoba, les coûts directs totaux attribuables aux effets sur la santé du tabagisme, de l'inactivité physique et d'un excédent de poids sont estimés à 490,3 millions de dollars, alors que les coûts indirects se situent à environ 1 113,8 millions de dollars, ce qui donne un fardeau économique annuel total de 1604,2 millions de dollars.

Ce fardeau global est passablement plus élevé pour les femmes (824,9 millions de dollars) que pour les hommes (779,3 millions de dollars). Si les coûts liés au tabagisme sont plus élevés chez les hommes (319,5 millions de dollars, contre 237,9 millions de dollars chez les femmes), le fardeau économique demeure plus élevé chez les femmes pour l'excédent de poids (417,7 millions de dollars contre 329,5 millions de dollars pour les hommes) et l'inactivité physique (169,3 millions de dollars contre 130,2 millions de dollars pour les hommes).

La figure 1 illustre le fardeau propre à chaque facteur de risque et fournit des données supplémentaires sur les éléments qui constituent les coûts indirects de la maladie. Le fardeau indirect de la mortalité prématurée liée au tabagisme domine (241,8 millions de dollars, soit 64,4\% des 375,4 millions de dollars en coûts indirects totaux pour ce facteur de risque), et il est aussi légèrement plus élevé que l'invalidité dans le cas de l'inactivité physique. L'inverse est vrai pour le surpoids et l’obésité : le fardeau économique de l'invalidité à court et à long termes liée à la maladie (311,5 millions de dollars) surpasse les coûts de la mortalité prématurée (218,6 millions de dollars) pour ce facteur de risque.

Le fardeau économique de l'excédent de poids ventilé selon notre méthode est plus important que celui du tabagisme. En fait, le fardeau économique du surpoids et de l'obésité combinés au Manitoba était de 747,2 millions de dollars (283,7 millions de dollars plus 463,5 millions de dollars) en 2008, ce qui excédait de $34 \%$ le fardeau économique lié au tabagisme (557,4 millions de dollars).

\section{Analyse de sensibilité}

Ce sont les estimations ponctuelles du RR qui ont été utilisées dans les résultats du modèle de base présentés plus haut. Or un certain degré d'incertitude accompagne ces estimations ponctuelles, comme en témoigne l'IC à $95 \%$. Pour évaluer les effets de cette incertitude sur les résultats, nous avons utilisé les limites inférieures et supérieures de l'IC à $95 \%$ pour le RR lié à chaque facteur de risque et chaque maladie dans le cadre d'une analyse de sensibilité. L'utilisation des limites inférieures a entraîné une réduction du fardeau économique total, le faisant passer de 1 604,2 millions de dollars à 1251,5 millions de dollars (soit $-22,0 \%$ ), alors que l'utilisation des limites supérieures a porté ce fardeau économique total à 1927,7 millions de dollars (ou $+20,2 \%$ ) (voir le tableau 4).

\section{Analyse}

La démarche analytique présentée ici a pris en compte la question du double compte des coûts au moment d'estimer le fardeau économique global des maladies chroniques liées à la présence de facteurs de risque multiples chez une même personne. Appliquée à la province du Manitoba, nous avons vu que cette démarche a conduit à envisager une réduction de $12,6 \%$ du fardeau économique global par rapport au fardeau total obtenu par la simple addition des coûts générés par chacun des principaux facteurs de risque.

Nous avons eu recours à une version élargie de la formule de base de la FER afin d'obtenir un résultat plus précis, notamment en tenant compte à la fois des complications que représente l'évaluation de la FER en présence d'un facteur de risque polytomique (soit le surpoids et 


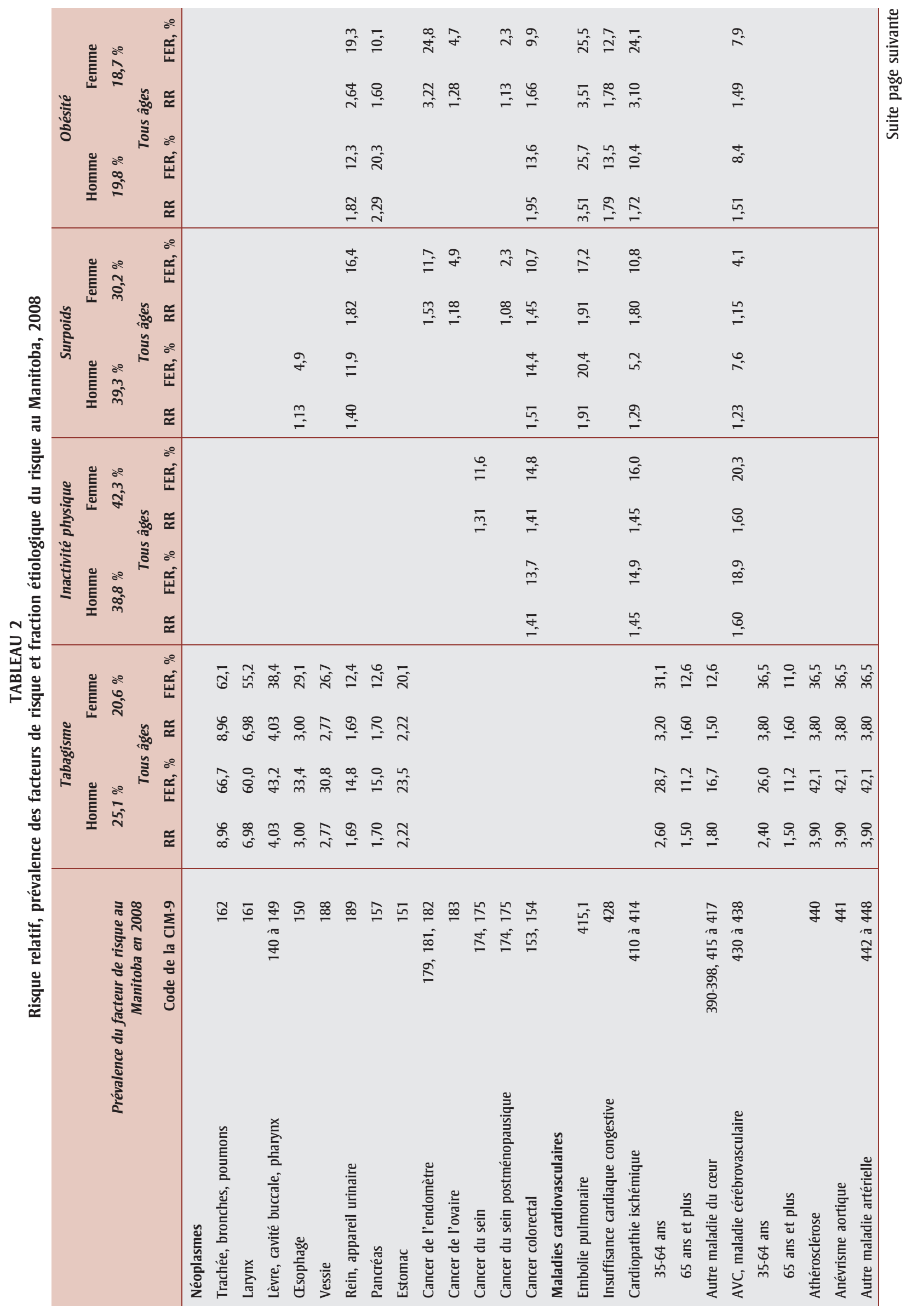




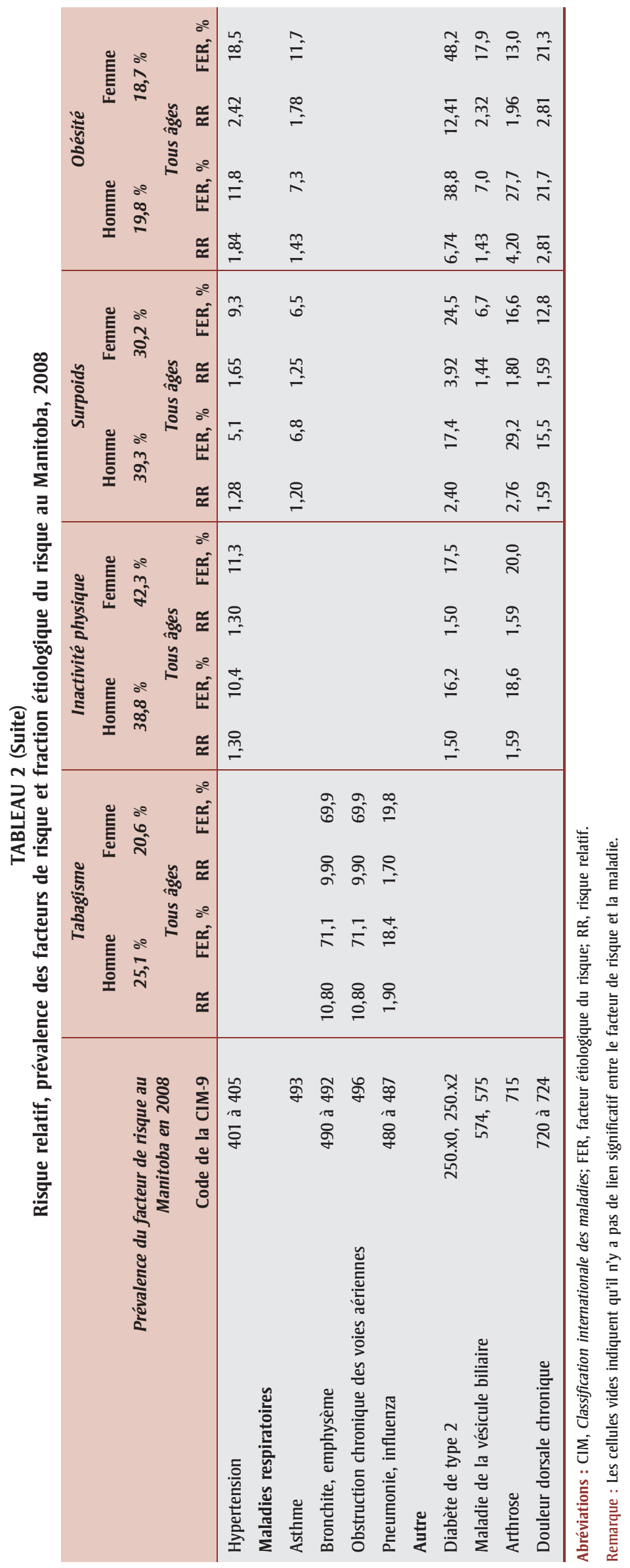

l'obésité) et de la possibilité que des facteurs de risque multiples soient présents chez une même personne.

L'analyse du fardeau économique lié aux facteurs de risque dans leur ensemble et à chacun des facteurs de risque particuliers que sont le tabagisme, l'inactivité physique et le surpoids ou l'obésité est la première étape de toute tentative visant à prévoir l'incidence économique potentielle de la mise en œuvre d'initiatives de prévention primaire.

Nous avons vu que, selon les estimations établies à l'aide des méthodes présentées dans cet article, le fardeau économique annuel total des facteurs de risque au Manitoba en 2008 serait de 1,6 milliard de dollars (490 millions de dollars en coûts directs et 1114 millions de dollars en coûts indirects).

Un autre résultat important, obtenu grâce à l'accès à des données sur le RR et la prévalence des facteurs de risque selon le sexe, est l'existence d'une différence entre les hommes et les femmes quant à leur contribution au fardeau économique total. Les coûts liés au tabagisme sont plus élevés pour les hommes, ce qui illustre en partie la prévalence toujours plus élevée de l'usage du tabac chez les hommes. À l'inverse, le fardeau économique lié à l'excédent de poids est supérieur chez les femmes, résultat qui semble anormal puisque la prévalence de l'obésité et (surtout) du surpoids est en fait plus élevée parmi les hommes. Cette anomalie s'explique, outre par le fardeau propre aux cancers gynécologiques chez les femmes, par le fait que le RR que représente chez elles un excédent de poids est plus élevé en ce qui concerne plusieurs maladies coûteuses comme le cancer du rein, la cardiopathie ischémique, l'hypertension et le diabète de type 2 (voir le tableau 2). Ainsi, la répartition globale selon le sexe du fardeau que représentent les principaux facteurs de risque modifiables a d'importantes conséquences pour la planification des stratégies de prévention et les messages de santé publique à transmettre à la population.

Notre analyse a également permis de confirmer l'émergence du surpoids et 
TABLEAU 3

Prévalence estimative des facteurs de risque, fardeau économique total dans un cadre multifactoriel et coûts ventilés par facteur de risque, Manitoba, 2008, selon le sexe : rajustements

\begin{tabular}{|c|c|c|c|c|c|c|c|c|}
\hline & $\begin{array}{l}\text { Pourcentage de } \\
\text { la population } \\
\text { avec FR (\%) }\end{array}$ & $\begin{array}{c}\text { Nombre de } \\
\text { personnes } \\
\text { avec FR }\end{array}$ & $\begin{array}{l}\text { Coût direct par } \\
\text { personne avec } \\
\text { FR (\$) }\end{array}$ & $\begin{array}{l}\text { Coût indirect } \\
\text { par personne } \\
\text { avec } F R(\$)\end{array}$ & $\begin{array}{c}\text { Coût total par } \\
\text { personne avec } \\
\text { FR (\$) }\end{array}$ & $\begin{array}{l}\text { Coût direct } \\
\text { du FR (MS) }\end{array}$ & $\begin{array}{l}\text { Coût indirect } \\
\text { du FR (MS) }\end{array}$ & $\begin{array}{l}\text { Coût total du } \\
\text { FR (MS) }\end{array}$ \\
\hline \multicolumn{9}{|l|}{ Hommes } \\
\hline Inactifs & 38,8 & 229124 & 180,2 & 388,2 & 568,4 & 41,3 & 88,9 & 130,2 \\
\hline Surpoids & 39,3 & 232251 & 141,6 & 418,1 & 559,7 & 32,9 & 97,1 & 130,0 \\
\hline Sous-total & & & & & & 235,0 & 544,3 & 779,3 \\
\hline \multicolumn{9}{|l|}{ Femmes } \\
\hline Fumeuses & 20,6 & 125013 & 636,1 & 1266,5 & 1902,7 & 79,5 & 158,3 & 237,9 \\
\hline Inactives & 42,3 & 257429 & 194,2 & 463,6 & 657,7 & 50,0 & 119,3 & 169,3 \\
\hline Surpoids & 30,2 & 183858 & 232,4 & 603,8 & 836,2 & 42,7 & 111,0 & 153,7 \\
\hline Fumeurs & 22,8 & 273473 & 665,5 & 1372,6 & 2038,1 & 182,0 & 375,4 & 557,4 \\
\hline Inactifs & 40,6 & 486553 & 187,6 & 428,1 & 615,7 & 91,3 & 208,3 & 299,6 \\
\hline Surpoids & 34,7 & 416109 & 181,7 & 500,2 & 681,9 & 75,6 & 208,1 & 283,7 \\
\hline Obésité & 19,2 & 230757 & 612,8 & 1395,7 & 2008,5 & 141,4 & 322,1 & 463,5 \\
\hline Total & & & & & & 490,3 & 1113,8 & 1604,2 \\
\hline
\end{tabular}

Abréviations : ESCC, Enquête sur la santé dans les collectivités canadiennes; FR, facteur de risque.

Remarque : Un rajustement a été effectué en fonction de certaines contraintes de données de l'ESCC et de la présence de facteurs de risque multiples chez une même personne.

FIGURE 1

Fardeau économique direct et indirect du tabagisme, de l'inactivité physique et du surpoids et de l'obésité, Manitoba, 2008

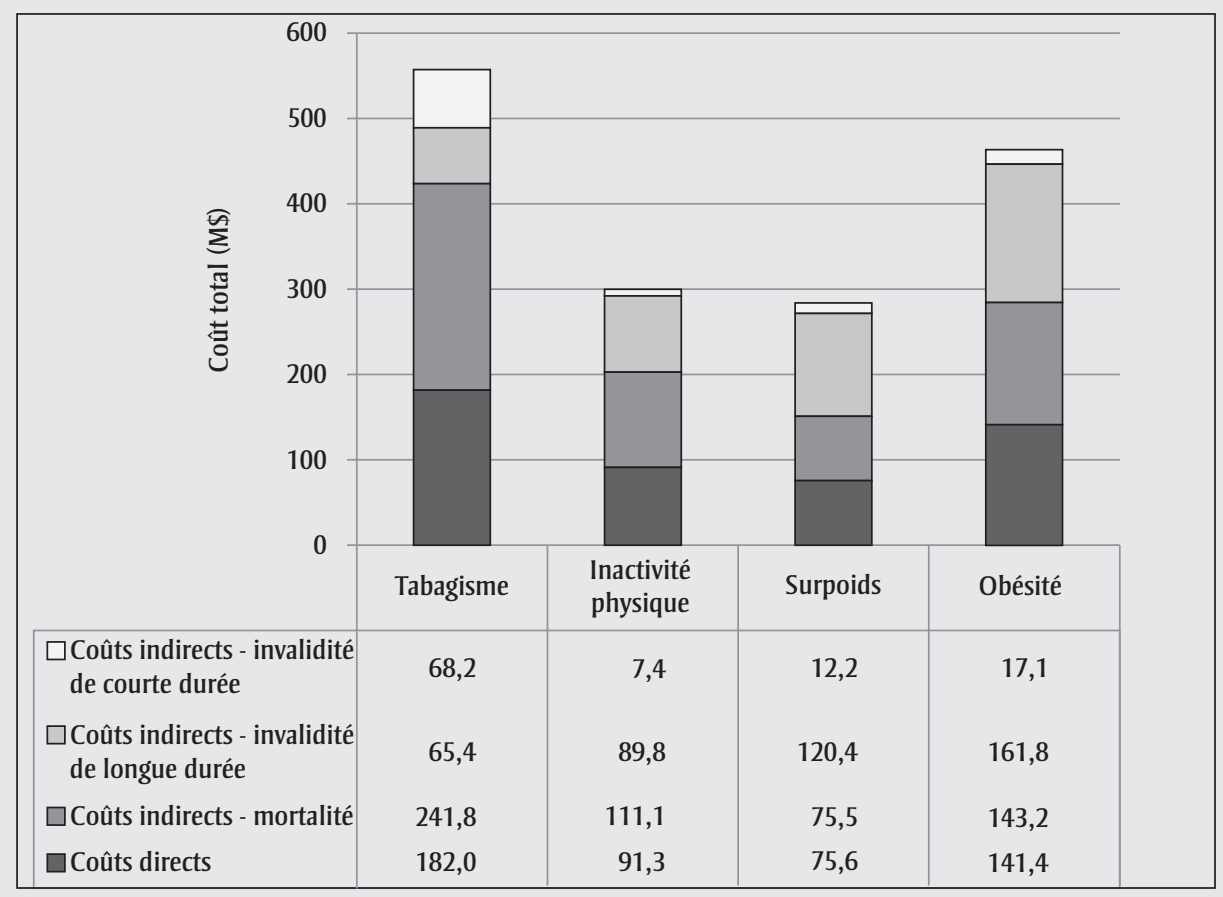

Remarque : Un rajustement a été effectué en fonction de certaines contraintes de données de l'ESCC et de la présence de facteurs de risque multiples chez une même personne. 
TABLEAU 4

Fardeau économique total estimatif, Manitoba, 2008, selon le sexe : analyse de sensibilité

\begin{tabular}{|c|c|c|c|c|c|}
\hline & \multicolumn{5}{|c|}{ Analyse de sensibilité } \\
\hline & $\begin{array}{c}\text { Meilleures } \\
\text { estimations du RR }\end{array}$ & $\begin{array}{c}\text { Estimation } \\
\text { inférieure du } R R\end{array}$ & Variance & $\begin{array}{c}\text { Estimation } \\
\text { supérieure du RR }\end{array}$ & Variance \\
\hline \multicolumn{6}{|l|}{ Hommes } \\
\hline Fumeurs & 319,5 & 266,3 & $-16,7$ & 363,0 & 13,6 \\
\hline Inactifs & 130,2 & 102,4 & $-21,4$ & 157,2 & 20,7 \\
\hline Surpoids & 130,0 & 95,2 & $-26,8$ & 159,0 & 22,3 \\
\hline Obésité & 199,5 & 147,5 & $-26,1$ & 248,3 & 24,5 \\
\hline Sous-total & 779,3 & 611,4 & $-21,5$ & 927,5 & 19,0 \\
\hline \multicolumn{6}{|l|}{ Femmes } \\
\hline Fumeuses & 237,9 & 203,3 & $-14,5$ & 272,3 & 14,5 \\
\hline Inactives & 169,3 & 129,7 & $-23,4$ & 206,3 & 21,9 \\
\hline Surpoids & 153,7 & 110,8 & $-27,9$ & 192,3 & 25,1 \\
\hline Obésité & 264,0 & 196,3 & $-25,6$ & 329,3 & 24,7 \\
\hline Sous-total & 824,9 & 640,1 & $-22,4$ & 1000,2 & 21,3 \\
\hline \multicolumn{6}{|l|}{ Ensemble } \\
\hline Fumeurs & 557,4 & 469,6 & $-15,8$ & 635,3 & 14,0 \\
\hline Inactifs & 299,6 & 232,1 & $-22,5$ & 363,5 & 21,4 \\
\hline Surpoids & 283,7 & 206,0 & $-27,4$ & 351,3 & 23,8 \\
\hline Obésité & 463,5 & 343,8 & $-25,8$ & 577,6 & 24,6 \\
\hline Total & 1604,2 & 1251,5 & $-22,0$ & 1927,7 & 20,2 \\
\hline
\end{tabular}

Abréviations : FR, facteur de risque; RR, risque relatif.

de l’obésité comme problème de santé publique, phénomène constaté également dans d'autres pays ${ }^{56,57}$ : le fardeau économique estimatif d'un excédent de poids

FIGURE 2

Chevauchement de l'exposition aux facteurs de risque au Canada, Enquête sur la santé dans les collectivités canadiennes, Cycle 1.1 (2000)

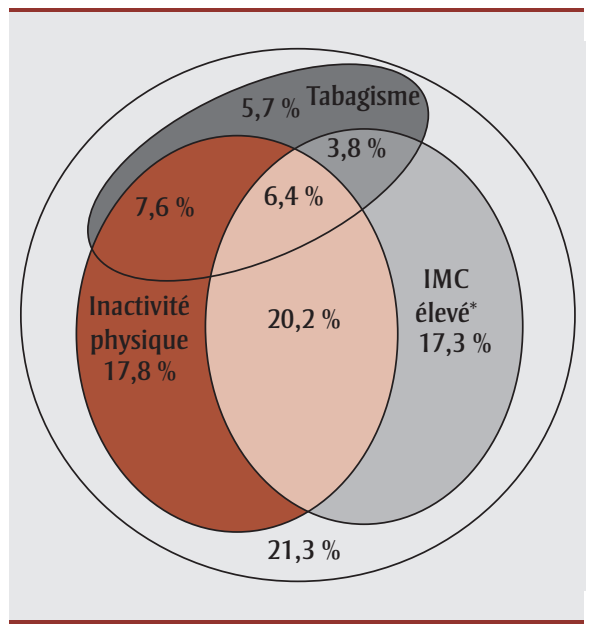

Source : Klein-Geltink et collab., Maladies chroniques au Canada, $2006^{59}$

Abréviation : IMC, indice de masse corporelle.

${ }^{*} \mathrm{IMC} \geq 25 \mathrm{~kg} / \mathrm{m}^{2}$. en 2008 au Manitoba (747,2 millions de dollars) est plus lourd que celui lié au tabagisme (557,4 millions de dollars). Bien que le fardeau économique lié au tabagisme surpasse toujours celui de l'obésité dans son sens le plus strict, l'ajout des effets du surpoids donne un facteur de risque combiné qui le classe au premier rang. Le projet mené au Royaume-Uni que nous avons mentionné plus haut ${ }^{26}$ est arrivé à des résultats similaires, c'est-à-dire que les coûts directs du surpoids et de l'obésité ( 5 milliards de livres) dépassent le fardeau total lié au tabagisme (3,3 milliards de livres) selon un écart semblable en proportion à celui établi ici pour le Manitoba. Cela dit, le point à partir duquel le surpoids est lié à une hausse importante des effets sur la santé est probablement plus élevé qu'un IMC de $25 \mathrm{~kg} / \mathrm{m}^{2}$ dans la population nordaméricaine, même s'il peut également être moins élevé au sein de certains groupes ethniques $^{58}$.

La qualité des résultats obtenus par l'analyse de la FER est inévitablement limitée par la qualité des intrants, particulièrement celle des données sur le RR et la prévalence. L'incidence des erreurs de précision susceptibles de s'être produites dans le cadre de notre projet a d'abord été atténuée en corrigeant les lacunes connues dans les données sur l'exposition aux facteurs de risque, obtenues dans le cadre des enquêtes menées régulièrement auprès de la population canadienne. Une variation dans les estimations régionales de la FER témoigne souvent d'une incertitude quant au niveau d'exposition au facteur de risque analysé ${ }^{33}$, d'où la nécessité de raffiner, autant que faire se peut, les données sur la prévalence.

L'estimation de tous les RR repose sur les données provenant de méta-analyses, lesquelles ont été rajustées dans la mesure du possible en fonction des facteurs confusionnels connus. Une analyse de la sensibilité au moyen de l'IC à $95 \%$ lié à chaque RR conclut à l'importance d'utiliser des estimations de RR robustes et précises.

Un rajustement (réduction) de 12,6\% pour les facteurs de risque qui se chevauchent chez certaines personnes semble-t-il valide? La figure 2 résume le degré de chevauchement possible entre les facteurs de risque confusionnels chez les Canadiens, selon les données de l'ESCC de $2000^{59}$. En faisant la somme des souscatégories pertinentes, $10,2 \%$ de la population est ainsi exposée au tabagisme et au surpoids ou à l'obésité, 26,6\%, au surpoids ou à l'obésité et à l'inactivité physique et 14,0\%, à l'inactivité physique et au tabagisme. Bien que le chevauchement d'un IMC élevé et de l'inactivité physique soit relativement élevé, la correction requise (pour éviter le double compte de l'incidence des maladies) a, en fait, été apportée dans ce cas aux données sur le RR de l'inactivité physique pour tenir compte du surpoids et de l'obésité ${ }^{16}$. Comparé à la proportion de la population exposée à des facteurs de risque multiples, le rajustement de $12,6 \% \mathrm{du}$ fardeau économique du Manitoba semble donc valide.

Malgré les efforts consentis pour estimer le fardeau économique avec une précision optimale, l'exercice présente toujours certaines limites, en partie à cause des hypothèses qu'il faut formuler afin d'intégrer de manière créative plusieurs 
sources de données compilées à divers points dans le temps. Par exemple, Anis et collab. ont présupposé, dans leur modèle de base lors de l'utilisation de données antérieures de l'ICIS et du rapport du FEMC, que « [traduction] la répartition des coûts entre chaque catégorie de coût n'a pas changé énormément entre 1998 et

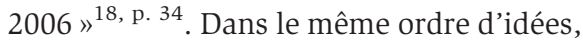
la méthode qui consiste à passer des coûts directs aux coûts indirects repose sur l'hypothèse que les rapports de coûts entre les diverses comorbidités sont les mêmes pour les coûts directs et indirects. En outre, les RR liés au tabagisme se fondent sur une comparaison entre fumeurs et non-fumeurs n'ayant jamais fumé, mais ne tiennent pas compte de l'intensité du tabagisme : des changements potentiels (réductions) dans l'intensité du tabagisme viendraient les modifier.

Les planificateurs de soins de santé de nombreux pays du monde aimeraient disposer d'une estimation raisonnable du fardeau économique des maladies attribuables à des facteurs de risque modifiables, car de telles données sont cruciales pour établir des priorités et soutenir les programmes de prévention primaire. En effet, il faut comprendre le fardeau économique fondamental lié à certains facteurs de risque avant de pouvoir élaborer une analyse de rentabilité convaincante en matière de prévention. Par exemple, nos résultats ont servi de catalyseur à la prise de mesures au Manitoba, en soutenant la mise en place d'un groupe de travail sur la prévention primaire, le lancement d'un défi de réduction des facteurs de risque aux politiciens provinciaux, la création d'une chaire de recherche en prévention primaire et l'obtention de subventions de la Fondation des maladies du cœur.

\section{Remerciements}

Cette étude a été financée par la Fondation des maladies du cœur du Manitoba, Action cancer Manitoba, l'Alliance pour la prévention des maladies chroniques au Canada et Health in Common.

\section{Références}

1. Omran AR. The epidemiologic transition. A theory of the epidemiology of population change. Milbank Mem Fund Q. 1971; 49(4):509-38.

2. Gaziano JM. Fifth phase of the epidemiologic transition: the age of obesity and inactivity. JAMA. 2010;303(3):275-6.

3. Klein S, Burke LE, Bray GA, Garbagnati F, Cappuccio FP, Scalfi L. Clinical implications of obesity with specific focus on cardiovascular disease: a statement for professionals from the American Heart Association Council on Nutrition, Physical Activity, and Metabolism: endorsed by the American College of Cardiology Foundation. Circulation. 2004;110(18): 2952-67.

4. Strazzullo P, D’Elia L, Cairella G, Garbagnati F, Cappuccio FP, Scalfi L. Excess body weight and incidence of stroke: meta-analysis of prospective studies with 2 million participants. Stroke. 2010;41(5):e418-26.

5. Abdullah A, Peeters A, de Courten M, Stoelwinder J. The magnitude of association between overweight and obesity and the risk of diabetes: a meta-analysis of prospective cohort studies. Diabetes Res Clin Pract. 2010;89(3):309-19.

6. Wang Y, Chen X, Song Y, Caballero B, Cheskin LJ. Association between obesity and kidney disease: a systematic review and meta-analysis. Kidney Int. 2008;73(1): 19-33.

7. Jiang L, Rong J, Wang Y, et collab. The relationship between body mass index and hip osteoarthritis: a systematic review and meta-analysis. Joint Bone Spine. 2011; 78(2):150-5.

8. Yang $\mathrm{P}$, Zhou $\mathrm{Y}$, Chen $\mathrm{B}$, et collab. Overweight, obesity and gastric cancer risk: results from a meta-analysis of cohort studies. Eur J Cancer. 2009;45(16):2867-73.

9. Larsson SC, Wolk A. Overweight, obesity and risk of liver cancer: a meta-analysis of cohort studies. Br J Cancer. 2007;97(7): 1005-8.
10. Moghaddam AA, Woodward M, Huxley R. Obesity and risk of colorectal cancer: a meta-analysis of 31 studies with 70,000 events. Cancer Epidemiol Biomarkers Prev. 2007;16(12):2533-47.

11. Warburton DE, Charlesworth S, Ivey A, Nettlefold L, Bredin SS. A systematic review of the evidence for Canada's Physical Activity Guidelines for Adults. Int J Behav Nutr Phys Act. 2010;7:39.

12. Friedenreich CM, Neilson HK, Lynch BM. State of the epidemiological evidence on physical activity and cancer prevention. Eur J Cancer. 2010;46(14):2593-604.

13. Glantz S, Gonzalez M. Effective tobacco control is key to rapid progress in reduction of non-communicable diseases. Lancet. 2012;379(9822):1269-71.

14. Withrow D, Alter DA. The economic burden of obesity worldwide: a systematic review of the direct costs of obesity. Obes Rev. 2011;12(2):131-41.

15. Katzmarzyk PT, Gledhill N, Shephard RJ. The economic burden of physical inactivity in Canada. CMAJ. 2000;163(11):1435-40.

16. Katzmarzyk PT, Janssen I. The economic costs associated with physical inactivity and obesity in Canada: an update. Can J Appl Physiol. 2004;29(1):90-115.

17. Birmingham CL, Muller JL, Palepu A, Spinelli JJ, Anis AH. The cost of obesity in Canada. CMAJ. 1999;160(4):483-8.

18. Anis AH, Zhang W, Bansback N, Guh DP, Amarsi Z, Birmingham CL. Obesity and overweight in Canada: an updated cost-ofillness study. Obes Rev. 2010;11(1):31-40.

19. Kaiserman MJ. Le coût du tabagisme au Canada, 1991. Maladies chroniques au Canada. 1997;18(1):15-22.

20. Moffatt E, Shack LG, Petz GJ, Sauvé JK, Hayward K, Colman R. The cost of obesity and overweight in 2005: a case study of Alberta, Canada. Can J Public Health. 2011;102(2):144-8.

21. Janssen I, Lam M, Katzmarzyk PT. Influence of overweight and obesity on physician costs in adolescents and adults in Ontario, Canada. Obes Rev. 2009;10(1): 51-7. 
22. Fine LJ, Philogene GS, Gramling R, Coups EJ, Sinha S. Prevalence of multiple chronic disease risk factors. 2001 National Health Interview Survey. Am J Prev Med. 2004;27(2 Suppl):18-24.

23. Ezzati M, Hoorn SV, Rodgers A et collab. Estimates of global and regional potential health gains from reducing multiple major risk factors. Lancet. 2003;362(9380): 271-80.

24. World Cancer Research Fund. Policy and action for cancer prevention - Food, nutrition, and physical activity: a global perspective - appendices [Internet]. Washington (DC): 2009 [consultation le 15 mai 2012]. PDF $(1,12 \mathrm{Mo})$ téléchargeable à partir du lien : http://www.dietandcancerreport.org /cancer_resource_center/downloads/chapters /pr/Appendix\%20A\%20and \%20B.pdf

25. Allender S, Foster C, Scarborough P, Rayner M. The burden of physical activity-related ill health in the UK. J Epidemiol Community Health. 2007;61(4):344-8.

26. Allender S, Rayner M. The burden of overweight and obesity-related ill health in the UK. Obes Rev. 2007;8(5):467-73.

27. Allender S, Balakrishnan R, Scarborough P, Webster P, Rayner M. The burden of smoking-related ill health in the UK. Tob Control. 2009;18(4):262-7.

28. Rayner M, Scarborough P. The burden of food related ill health in the UK. J Epidemiol Community Health. 2005;59(12): 1054-7.

29. Balakrishnan R, Allender S, Scarborough P, Webster P, Rayner M. The burden of alcohol-related ill health in the United Kingdom. J Public Health (Oxf). 2009; 31(3):366-73.

30. Scarborough P, Bhatnagar P, Wickramasinghe KK, Allender S, Foster C, Rayner M. The economic burden of ill health due to diet, physical inactivity, smoking, alcohol and obesity in the UK: an update to 2006-07 NHS costs. J Public Health (Oxf). 2011;33(4):527-35.
31. Statistique Canada. Série " Perspective géographique ", Recensement de 2011 : Province du Manitoba [Internet]. Ottawa (Ont.) : Statistiques Canada; 2012 [consultation le 15 mai 2012]. Consultable à la page : http://www12.statcan.gc.ca/census -recensement/2011/as-sa/fogs-spg/Facts-pr -fra.cfm?LANG $=$ Fra\&GK $=$ PR\&GC $=46$

32. Affaires autochtones et Développement du Nord Canada. Les Premières nations au Manitoba [Internet]. Ottawa (Ont.) : Affaires autochtones et Développement du Nord Canada; 2012 [consultation le 15 mai 2012]. Consultable à la page : http://www .aadnc-aandc.gc.ca/fra/1100100020400 /1100100020404

33. Walter SD. Calculation of attributable risks from epidemiological data. Int J Epidemiol. 1978;7(2):175-82.

34. Guh DP, Zhang W, Bansback N, Amarsi Z, Birmingham CL, Anis AH. The incidence of co-morbidities related to obesity and overweight: a systematic review and metaanalysis. BMC Public Health. 2009;9:88.

35. Gandini S, Botteri E, Iodice S et collab. Tobacco smoking and cancer: a metaanalysis. Int J Cancer. 2008;122(1):155-64.

36. Thun MJ, Apicella LF, Henley SJ. Smoking vs other risk factors as the cause of smoking-attributable deaths: confounding in the courtroom. JAMA. 2000;284(6): 706-12.

37. Wendel-Vos GC, Schuit AJ, Feskens EJ, et collab. Physical activity and stroke. A metaanalysis of observational data. Int $\mathrm{J}$ Epidemiol. 2004;33(4):787-98.

38. Wolin KY, Yan Y, Colditz GA, Lee IM. Physical activity and colon cancer prevention: a meta-analysis. $\mathrm{Br} \mathrm{J}$ Cancer. 2009;100(4):611-6.

39. Harriss DJ, Atkinson G, George K, et collab. Lifestyle factors and colorectal cancer risk (1): systematic review and meta-analysis of associations with body mass index. Colorectal Dis. 2009;11(6):547-63.

40. Walter SD. Local estimates of population attributable risk. J Clin Epidemiol. 2010;63(1):85-93.
41. Health in common. Youth health survey report 2009: students in Manitoba (grades 9-12) [Internet]. Winnipeg (MB): Partners in Planning for Healthy Living; 2009 [consultation le 15 mai 2012]. PDF (2,0 Mo) téléchargeable à partir du lien : http://www .healthincommon.ca/wp-content/uploads /Youth-Health-Survey-Report-2009.pdf

42. Statistique Canada. Tableau 105-05011 : Profil d'indicateurs de la santé, estimations annuelles, selon le groupe d'âge et le sexe, Canada, provinces, territoires, régions sociosanitaires (limites de 2011) et groupes de régions homologues [Internet]. Ottawa (Ont.) : Statistique Canada; [consultation le 15 mai 2012]. Consultable à la page : http ://www5.statcan.gc.ca/cansim/a05;jsessionid $=12045 \mathrm{FD} 63 \mathrm{BEAA20647AE3E032736D95E? \textrm {id }}$ $=1050501 \&$ retrLang $=$ fra\&lang $=$ fra

43. Craigie AM, Lake AA, Kelly SA, Adamson AJ, Mathers JC. Tracking of obesity-related behaviours from childhood to adulthood: a systematic review. Maturitas. 2011;70(3): 266-84.

44. Statistique Canada. Recensement de 2006 : Peuples autochtones du Canada en 2006 : Inuits, Métis et Premières nations, Recensement de 2006 : résultats [Internet]. Ottawa (Ont.) : Statistique Canada; 2006 [consultation le 15 mai 2012]. Consultable à la page : http: //www12.statcan.ca/census-recensement /2006/as-sa/97-558/index-fra.cfm

45. Regional health survey, 2002/03 [Internet]. Winnipeg (MB): Manitoba First Nations; 2005 [consultation le 15 mai 2012]. Consultable à la page : http://www.manitobachiefs .com

46. Hanley JA. A heuristic approach to the formulas for population attributable fraction. J Epidemiol Community Health. 2001;55(7):508-14.

47. Steenland K, Armstrong B. An overview of methods for calculating the burden of disease due to specific risk factors. Epidemiology. 2006;17(5):512-9.

48. Healton CG, Vallone D, McCausland KL, Xiao H, Green MP. Smoking, obesity, and their co-occurrence in the United States: cross sectional analysis. Br Med J. 2006; 333(7557):25-6. 
49. Freedman DM, Sigurdson AJ, Rajaraman P, Doody MM, Linet MS, Ron E. The mortality risk of smoking and obesity combined. Am J Prev Med. 2006;31(5):355-62.

50. Katzmarzyk PT, Janssen I, Ardern CI. Physical inactivity, excess adiposity and premature mortality. Obes Rev. 2003; 4(4): 257-90.

51. Lee DC, Sui X, Blair SN. Does physical activity ameliorate the health hazards of obesity? Br J Sports Med. 2009; 43(1): 49-51.

52. Tarricone R. Cost-of-illness analysis: what room in health economics? Health Policy. 2006;77:51-63.

53. Institut canadien d'information sur la santé. Tendances des dépenses nationales de santé, 1975 à 2011 [Internet]. Ottawa (Ont.) : ICIS; 2011 [consultation le $1^{\mathrm{er}}$ juin 2012]. PDF (3,54 Mo) téléchargeable à partir de la page : https://secure.cihi.ca /estore/productFamily.htm?locale $=$ fr\&pf $=$ PFC1671

54. Santé Canada. Le fardeau économique de la maladie au Canada 1998 [Internet]. Ottawa (Ont.) : Santé Canada; 2002 [consultation le 15 mai 2012]. Consultable à la page : http://www.phac-aspc.gc.ca/ebic-femc/ebic -femc98/index-fra.php

55. Institut canadien d'information sur la santé. Hospital morbidity database, 2000/01 Tabular Results [Internet]. Ottawa (Ont.) : Institut canadien d'information sur la santé; 2002 [consultation le $1^{\mathrm{er}}$ juin 2012]. PDF (2,19 Mo) téléchargeable à partir du lien : https://secure.cihi.ca/free_products /HospitalMorbidityTabularReports2000-2001 .pdf

56. Jia H, Lubetkin EI. Trends in qualityadjusted life-years lost contributed by smoking and obesity. Am J Prev Med. 2010;38(2):138-44.

57. Hoad V, Somerford P, Katzenellenbogen J. High body mass index overtakes tobacco as the leading independent risk factor contributing to disease burden in Western Australia. Aust NZ J of Publ Heal. 2010;34(2):214-5.
58. Krueger H, Williams D, Kaminsky B, McLean D. The health impact of smoking and obesity and what to do about it. Toronto (Ont.): University of Toronto Press; 2007.

59. Klein-Geltink JE, Choi BC, Fry RN. Multiple exposures to smoking, alcohol, physical inactivity and overweight: prevalences according to the Canadian Community Health Survey Cycle 1.1. Chronic Dis Can. 2006;27(1):25-33 